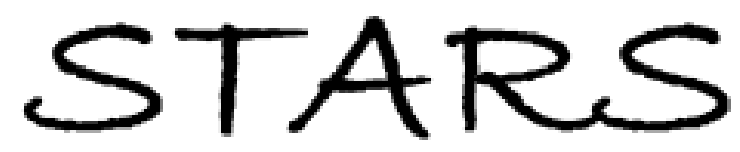

University of Central Florida

STARS

$1-1-2009$

\title{
Superconducting properties of a boson-exchange model of doped graphene
}

\author{
V. M. Loktev \\ V. Turkowski \\ University of Central Florida
}

Find similar works at: https://stars.library.ucf.edu/facultybib2000 University of Central Florida Libraries http://library.ucf.edu

This Article is brought to you for free and open access by the Faculty Bibliography at STARS. It has been accepted for inclusion in Faculty Bibliography 2000 s by an authorized administrator of STARS. For more information, please contactSTARS@ucf.edu.

\section{Recommended Citation}

Loktev, V. M. and Turkowski, V., "Superconducting properties of a boson-exchange model of doped graphene" (2009). Faculty Bibliography 2000s. 1827.

https://stars.library.ucf.edu/facultybib2000/1827

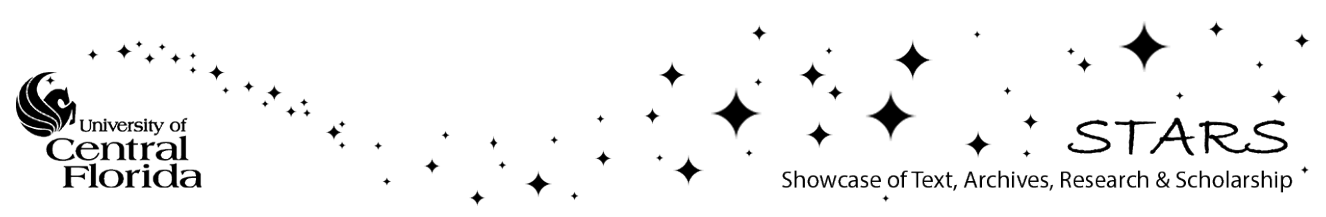




\section{Superconducting properties of a boson- exchange model of doped graphene}

Cite as: Low Temp. Phys. 35, 632 (2009); https://doi.org/10.1063/1.3224719

Published Online: 01 December 2009

(d) V. M. Loktev, and V. Turkowski

\section{ARTICLES YOU MAY BE INTERESTED IN}

Strain-tunable Josephson current in graphene-superconductor junction

Applied Physics Letters 103, 182603 (2013); https://doi.org/10.1063/1.4828567

Graphene-based ferromagnetic superconductors

Applied Physics Letters 101, 252602 (2012); https://doi.org/10.1063/1.4772507

Thermal transport properties of graphene-based ferromagnetic/singlet superconductor/ ferromagnetic junctions

Journal of Applied Physics 107, 123916 (2010); https://doi.org/10.1063/1.3452364

LOW TEMPERATURE TECHNIQUES OP『ICAL CAVITY PHVSICS MITIGATING THERMAL \& VIBRATIONAL NOISE

\section{DOWNLOAD THE WHITE PAPER}




\title{
Superconducting properties of a boson-exchange model of doped graphene
}

\author{
V. M. Loktev ${ }^{\text {a) }}$ \\ Bogolyubov Institute for Theoretical Physics, Metrologichna St. 14-b, Kiev 03680, Ukraine \\ V. Turkowski
}

Department of Physics and NanoScience Technology Center, University of Central Florida, Orlando, Florida 32816

(Submitted February 16, 2009)

Fiz. Nizk. Temp. 35, 805-811 (August-September 2009)

\begin{abstract}
We study the superconducting properties of a doped one-layer graphene by using a model in which the interparticle attraction is caused by a boson (phonon-like) excitations. We study the dependence of the superconducting gap $\Delta$ and the mean-field critical temperature $T_{c}^{M F}$ on the carrier density, attraction strength, and characteristic (Debye) bosonic frequency. In addition, we study the temperature-carrier density phase diagram of the model by taking into account the thermal fluctuations of the order parameter. We show that the fluctuations result in a significant suppression of $T_{c}^{M F}$, such that the real (Berezinskii-Kosterlitz-Thouless) critical temperature $T_{c}$ is much lower than $T_{c}^{M F}$. The region $T_{c}<T<T_{c}^{M F}$ is characterized by a finite density of states at the Fermi level (the pseudogap phase). We show that the width of the temperature interval of the pseudogap phase depends strongly on the model parameters-carrier concentration, attraction amplitude, and boson frequency. (C) 2009 American Institute of Physics. [doi:10.1063/1.3224719]
\end{abstract}

\section{INTRODUCTION}

The possibility of superconductivity in graphene is one of the most interesting and important problems of the modern theory of superconductivity. This problem is a part of the more general problem of understanding the physical, especially the electronic, properties of graphene, which is one of the most important basic units of carbon-based structures. These structures have a great potential to be used in modern nanotechnologies, especially due to strong and flexible carbon bonds. The single layer of graphene can be considered as the basis for two-dimensional (one-to several-layer graphene), one-dimensional (carbon nanotubes and nanoribbons), and zero-dimensional (fullerene molecules, graphene flakes, etc.) structures. Naturally, one needs to understand the low-temperature properties of such systems, when it is possible that the ground state of the system is the superconducting state. Moreover, the superconducting properties of graphene demostrate many interesting applications. During the last years, great progress has been made in understanding some of the electronic properties of graphene (for overview and references, see Ref. 1).

Evidences of superconductivity in graphite and graphitesulfur structures have been found experimentally. ${ }^{2-5}$ Theoretical studies of superconductivity in graphene were performed in Refs. 6-14. Since the Fermi surface of undoped graphene consists of Dirac points, the quantum-critical-point scenario of superconductivity in this case was analyzed in Refs. 6 and 8. It was found that in the undoped case, superconductivity takes place when the coupling is greater than a critical value. Superconductivity in graphene within different scenarios, like the phonon, plasmon, ${ }^{9}$ resonant-valence-bond, and density-wave scenario, was considered in Refs. 6, 10, and 11. In the doped case, when the Fermi surface is finite, the superconductivity can take place at any value of coupling. The doped case was analyzed within the framework of different models in Refs. 6, 7, 9, 10, 12, and 13. In particular, a simple model with an electron-electron attraction which is defined by the amplitude and the range (or the Debye-like cutoff energy $\omega_{0}$ in the boson-exchange model) was considered in Ref. 12. The authors solved the coupled system of the BCS gap equation and the particle number equation, in order to analyze the coupling dependence and doping dependence of the superconductivity. The possibility of strong enhancement of superconductivity with the critical temperature up to $T_{c}$ $\sim 10 \mathrm{~K}$, due to the van Hove singularities in the electron density of states in graphene at energies of order $3 \mathrm{eV}$ with respect to the band bottom was discussed in Ref. 13. One needs to realize in practice such highly doped samples of graphene in order to check the possibility of the strong enhancement of superconductivity. The possibility of superconductivity in the papers mentioned above was analyzed within the mean-field approximation, when the fluctuations were neglected. Such fluctuations cannot be neglected in 2D systems, since they can significantly modify the properties of the system. In particular, the thermal fluctuations in a twodimensional system lead to a significant reduction of the mean-field superconducting critical temperature $T_{c}^{M F}$ (Refs. 15 and 17). In this case, the real critical temperature is the Berezinskii-Kosterlitz-Thouless (BKT) temperature $T_{c}$ $\equiv T_{B K T}<T_{c}^{M F}$, below which the order parameter is algebraically ordered. Recently, we have studied the superconducting properties of the model considered in Ref. 12 in the case of single- and double-layer graphene by taking into account the thermal fluctuations. ${ }^{14}$ We have shown that the fluctuations lead to a drastic reduction of the superconducting critical temperature in both cases. We have also shown that at temperatures $T_{c}<T<T_{c}^{M F}$ in both systems the fluctuations of the order parameter produce so-called pseudogap phase with a reduced DOS. In that paper, we have considered the limit $\omega_{0}=\infty$, which corresponds to the case of local attraction. 
Here we consider the case of superconductivity for bosonexchange electron-electron attraction and show that the behavior of the system depends strongly on the bosonic energy (Debye frequency in the phonon case). The results obtained in this paper can lead to a further clarification of the mechanism of possible superconductivity in graphene.

We dedicate this paper to an outstanding person, the organizer and first Director of the Institute for Low Temperature Physics and Engineering, Prof. B. I. Verkin, for whom the physics of superconductivity was one of the main research interests.

\section{THE MODEL}

The effective superconducting Hamiltonian of doped single-layer graphene has the following form:

$$
\begin{aligned}
H(\tau)= & \sum_{\sigma} \psi_{\sigma}^{\dagger}(\tau, \mathbf{r})[\varepsilon(-i \nabla)-\mu] \psi_{\sigma}(\tau, \mathbf{r}) \\
& -\frac{1}{2} \int d \mathbf{r}_{1} \int d \mathbf{r}_{2} \psi_{\uparrow}^{\dagger}\left(\tau, \mathbf{r}_{2}\right) \psi_{\downarrow}^{\dagger}\left(\tau, \mathbf{r}_{1}\right) V\left(\mathbf{r}_{1}\right. \\
& \left.-\mathbf{r}_{2}\right) \psi_{\downarrow}\left(\tau, \mathbf{r}_{1}\right) \psi_{\uparrow}\left(\tau, \mathbf{r}_{2}\right),
\end{aligned}
$$

where $\psi_{\sigma}^{\dagger}(\tau, \mathbf{r})$ and $\psi_{\sigma}(\tau, \mathbf{r})$ are the fermionic operators of creation and annihilation of a quasiparticle on the site $\mathbf{r}$ at time $\tau$ with spin $\sigma=\uparrow \downarrow ; \varepsilon(-i \nabla)$ and $\mu$ are the free-fermion dispersion relation and the chemical potential, and $V\left(\mathbf{r}_{1}\right.$ $\left.-\mathbf{r}_{2}\right)$ is the interparticle attraction, respectively. The energy spectrum $\varepsilon(\mathbf{k})$ around the Dirac points can be approximated as $\varepsilon^{ \pm}(\mathbf{k})= \pm v_{F}|\mathbf{k}|$, where $v_{F}=3 \mathrm{ta} / 2 \sim 10^{6} \mathrm{~m} / \mathrm{s}$ is the Fermi velocity, $t=2.8 \mathrm{eV}$ is the nearest-neighbor hopping, and $a$ $=1.42 \AA$ is the distance between the nearest carbon atoms. In the undoped case, the band $\varepsilon^{-}(\mathbf{k})$ is filled. For definiteness, we consider the electron-doped case with the conduction band $\varepsilon^{+}(\mathbf{k}) \equiv \varepsilon(\mathbf{k})$.

We approximate the superconducting attraction by the following interparticle potential:

$$
V_{\mathbf{p}}=V_{0} \theta\left(\omega_{0}-|\varepsilon(\mathbf{p})-\mu|\right)
$$

where $V_{0}$ is the amplitude of the attraction and $\omega_{0}$ is the bosonic (Debye-like) cutoff frequency, which corresponds to a half of the BCS attraction shell around the Fermi energy surface, which, in most cases, is proportional to $\mu$ (see discussion below). We shall study the dependences of the superconducting properties of the model on $V_{0}$ and $\omega_{0}$ at different values of the doping, since knowledge of such dependences can help establish the source of possible superconducting attraction in graphene (the coupling energy and boson frequency in the case of a bosonic mechanism of superconductivity). We shall consider the isotropic $s$-wave pairing, when the gap $\Delta(\mathbf{k})$ is momentum independent; however, the results obtained below will remain qualitatively the same for more complicated interactions and different symmetries of the order parameter (see, e.g., Ref. 17).

\section{THE MEAN-FIELD APPROXIMATION}

In order to study the superconducting properties of the system, we shall consider the partition function

$$
\begin{aligned}
Z= & \int D \psi^{\dagger} D \psi \exp [ \\
& \left.-\int_{0}^{1 / T} d \tau\left(\sum_{\sigma} \int d \mathbf{r} \psi_{\sigma}^{\dagger}(\tau, \mathbf{r}) \partial_{\tau} \psi_{\sigma}(\tau, \mathbf{r})+H(\tau)\right)\right],
\end{aligned}
$$

which can be obtained by performing the path integration over the fermionic fields. To find $Z$, one can introduce the Nambu spinor operators:

$$
\Psi(\tau, \mathbf{r})=\left(\begin{array}{c}
\psi_{\uparrow}(\tau, \mathbf{r}) \\
\psi_{\downarrow}^{\dagger}(\tau, \mathbf{r})
\end{array}\right), \quad \Psi^{\dagger}(\tau, \mathbf{r})=\left(\psi_{\uparrow}^{\dagger}(\tau, \mathbf{r}), \psi_{\downarrow}(\tau, \mathbf{r})\right) .
$$

We use the Hubbard-Stratonovich transformation in order to decouple the four-fermion term in the Hamiltonian (see Eq. (1)). In this case, the partition function is equivalent to

$$
Z=\int D \Psi^{\dagger} D \Psi D \Phi^{\dagger} D \Phi e^{-S\left(\Psi^{\dagger}, \Psi, \Phi^{\dagger}, \Phi\right)},
$$

where

$$
\begin{aligned}
S\left(\Psi^{\dagger}, \Psi, \Phi^{\dagger}, \Phi\right)= & \int_{0}^{1 / T} d \tau \int d \mathbf{r}_{1} \int d \mathbf{r}_{2}\left\{\delta \left(\mathbf{r}_{1}\right.\right. \\
& \left.-\mathbf{r}_{2}\right) \sum_{\sigma} \psi_{\sigma}^{\dagger}\left(\tau, \mathbf{r}_{1}\right)\left[\partial_{\tau}+\tau_{z}\left(\varepsilon\left(\nabla_{\mathbf{r}_{2}}\right)\right.\right. \\
& -\mu)] \psi_{\sigma}\left(\tau, \mathbf{r}_{2}\right)+\frac{1}{2} \frac{\left|\Phi\left(\mathbf{r}_{1}, \mathbf{r}_{2}\right)\right|^{2}}{V\left(\mathbf{r}_{1}-\mathbf{r}_{2}\right)} \\
& -\frac{1}{2} \Psi^{\dagger}\left(\tau, \mathbf{r}_{1}\right) \tau_{+} \Psi\left(\tau, \mathbf{r}_{2}\right) \Phi\left(\tau, \mathbf{r}_{1}, \mathbf{r}_{2}\right) \\
& \left.-\frac{1}{2} \Phi^{\dagger}\left(\tau, \mathbf{r}_{1}, \mathbf{r}_{2}\right) \Psi\left(\tau, \mathbf{r}_{1}\right) \tau=\Psi\left(\tau, \mathbf{r}_{2}\right)\right\},
\end{aligned}
$$

is the effective action, and $\tau_{ \pm}=\frac{1}{2}\left(\tau_{x} \pm i \tau_{y}\right)$ and $\tau_{z}$ are the Pauli matrices. In Eq. (6), $\Phi\left(\tau, \mathbf{r}_{1}, \mathbf{r}_{2}\right) \sim V\left(\mathbf{r}_{1}\right.$ $\left.-\mathbf{r}_{2}\right) \psi_{\downarrow}\left(\tau, \mathbf{r}_{1}\right) \psi_{\uparrow}\left(\tau, \mathbf{r}_{2}\right)$ is a complex function, which has the physical meaning of the superconducting order parameter. After integration over the Nambu spinors in Eq. (5) one can get the expression for the thermodynamic potential $\Omega\left(\Phi^{\dagger}, \Phi\right)$ :

$$
\mathrm{e}^{-\Omega\left(\Phi^{\dagger}, \Phi\right) / T}=\int D \Psi^{\dagger} D \Psi \mathrm{e}^{-S\left(\Psi^{\dagger}, \Psi, \Phi^{\dagger}, \Phi\right)}
$$

In the case of the mean-field approximation, we assume that $\Phi\left(\tau, \mathbf{r}_{1}, \mathbf{r}_{2}\right)$ is independent of momentum and time in the momentum representation, and is equal to $\Delta(\tau, \mathbf{k}) \equiv \Delta$ $=$ const. In order to find the equations for the superconducting gap $\Delta$ and the chemical potential, one needs to minimize $\Omega(\Delta)$ with respect to $\Delta$ together with the particle-number equation $(1 / V) \partial \Omega / \partial \mu=-n_{f}$ ( $V$ is the volume of the system). This gives:

$$
1=\frac{1}{2} \sum_{\mathbf{k}} V(\mathbf{k}) \tanh \left(\frac{E(\mathbf{k})}{2 T}\right) \frac{1}{E(\mathbf{k})},
$$




$$
n_{f}=2 \sum_{\mathbf{k}}\left[1-\tanh \left(\frac{E(\mathbf{k})}{2 T}\right) \frac{\varepsilon(\mathbf{k})-\mu}{E(\mathbf{k})}\right],
$$

where $E(\mathbf{k})=\sqrt{(\varepsilon(\mathbf{k})-\mu)^{2}+\Delta^{2}}$ is the quasiparticle spectrum in the superconducting state. The solution of the system of equations (8) and (9) gives the doping and temperature dependence of $\Delta$ and also the doping dependence of the meanfield critical temperature $T_{c}^{M F}$ (at $\left.\Delta=0\right)$.

At $T=0$ the integration in Eqs. (8) and (9) can be easily performed, and one gets the following system of equations for the superconducting gap and chemical potential as functions of the Fermi energy $\varepsilon_{F}=v_{F} \sqrt{8 \pi n_{f}}$, coupling $\lambda$ $=V_{0} / 4 \pi v_{F}^{2}$ and bosonic frequency $\omega_{0}$ :

$$
\begin{aligned}
\frac{1}{\lambda}= & \theta\left(\omega_{0}-\mu\right)\left\{\sqrt{\omega_{0}^{2}+\Delta^{2}}-\sqrt{\mu^{2}+\Delta^{2}}\right. \\
& \left.+\mu \ln \left[\left(\omega_{0}+\sqrt{\omega_{0}^{2}+\Delta^{2}}\right)\left(\mu+\sqrt{\mu^{2}+\Delta^{2}}\right) / \Delta^{2}\right]\right\} \\
& +\theta\left(\mu-\omega_{0}\right) 2 \mu \ln \left(\left(\omega_{0}+\sqrt{\omega_{0}^{2}+\Delta^{2}}\right) / \Delta\right), \\
\frac{\varepsilon_{F}^{2}}{4}= & -\frac{\Delta^{2}}{2}+\mu^{2}+3 \mu \sqrt{\mu^{2}+\Delta^{2}}+\Delta^{2} \ln ((W-\mu \\
& \left.\left.+\sqrt{(W-\mu)^{2}+\Delta^{2}}\right)\left(\mu+\sqrt{\mu^{2}+\Delta^{2}}\right) / \Delta^{2}\right),
\end{aligned}
$$

where $\theta(x)$ is the step function and we have assumed that the bandwidth $W$ is much greater than $\mu, \omega_{0}$, and $\Delta$. One can find an approximate analytical solution of the equation for the zero-temperature superconducting gap:

$$
\Delta \simeq \sqrt{2 \omega_{0} \mu} \mathrm{e}^{-\left(1 / \lambda-\omega_{0}\right) / 2 \mu} \theta\left(\omega_{0}-\mu\right)+2 \omega_{0} \mathrm{e}^{-1 / 2 \mu \lambda} \theta\left(\mu-\omega_{0}\right) .
$$

In deriving this result we assumed that $\omega_{0} \gg \Delta$. One can show that at zero doping, $\Delta=0$ when $\lambda<1 / \omega_{0}$. In other words, in this case there is a critical value of the coupling below which there is no pairing. At finite doping, superconductivity takes place at any finite value of the coupling (see Ref. 12). One can compare the result of Eq. (12) with the corresponding expression for the two-dimensional system with parabolic dispersion:

$$
\Delta \simeq 2 \sqrt{\omega_{0}}\left[\sqrt{\varepsilon_{F}} \theta\left(\omega_{0}-\mu\right)+\sqrt{\omega_{0}} \theta\left(\mu-\omega_{0}\right)\right] \mathrm{e}^{-4 \pi / m V_{0}} .
$$

(see, e.g., Ref. 17). There are several important differences between the results (12) and (13). Namely, i) in the case of graphene, at low doping the gap is exponentially suppressed when $\mu$ is less than $\frac{1}{2}\left((1 / \lambda)-\omega_{0}\right)$ (Figs. 1 and 2$)$. This suppression is due to the factor in the exponent, which is proportional to the single-particle density of states (DOS) on the Fermi level. In the case with parabolic dispersion, the DOS on the Fermi level is a constant. ii) Another consequence of the difference of the density of states is increasing of the gap with growth of the carrier density. In the case of Eq. (13), the gap is carrier density independent at large doping, approximately when $\mu>\omega_{0}$. The doping dependence of the gap was analyzed by assuming that the doping is proportional to the chemical potential. Strictly speaking, in the two-dimensional system this is correct only for parabolic dispersion at large doping. As follows from Eq. (11), in our case the particle density is proportional to $\mu^{2}$ when $\mu \gg \Delta, \Delta \ln W$, which is true when the doping is rather large and the coupling is

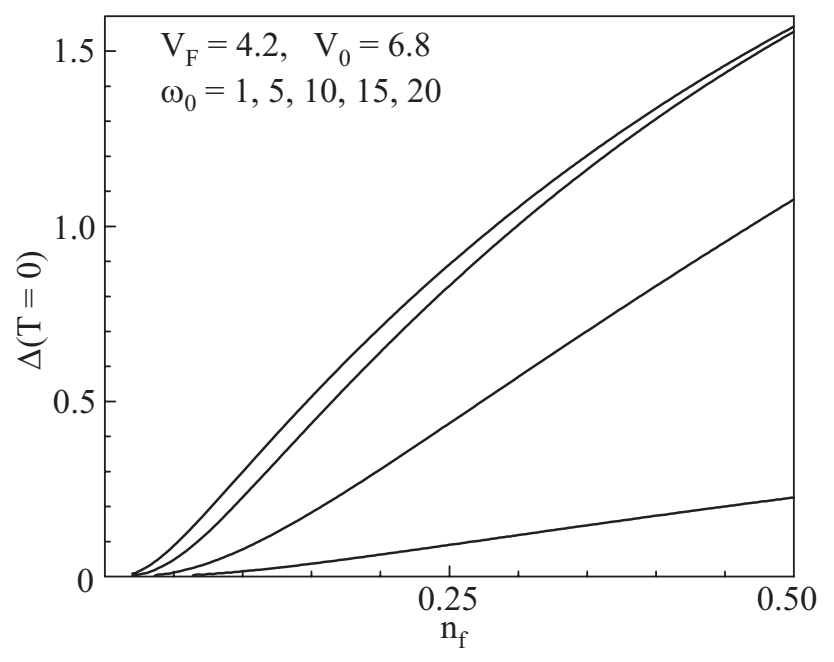

FIG. 1. The doping dependence of the zero-temperature gap at different values of the bosonic frequency $\omega_{0}$. Here and in other figures all parameters are given in $\mathrm{eV}$, and the carrier density is divided by two.

weak. In other cases, the relation between $n_{f}$ and $\mu$ is more complicated. Generally speaking, one can also obtain the crossover from superconductivity to superfluidity with decreasing doping and/or increasing the coupling, when the chemical potential becomes negative (for an overview, see Ref. 17). However, in actual paper we consider the superconductivity scenario only, by assuming that $\mu$ is positive (see Eq. (2), where the interaction is defined inside the belt $-\omega_{0}$ $<\varepsilon<\omega_{0}$ around the "Fermi energy"). The dependences of $T_{c}^{M F}$ on $n_{f}, V_{0}$, and $\omega_{0}$ are the same as those of $\Delta(T=0)$ (Figs. 3 and 4). Moreover, we have found that the ratio $2 \Delta(T$ $=0) / T_{c}^{M F}$ is close to the BCS value 3.52. In the next Section, we demonstrate that the real $T_{c}$ is much lower than $T_{c}^{M F}$ due to the thermal fluctuations in the two-dimensional system.

\section{FLUCTUATIONS}

In order to study the fluctuation effects in the system, we represent the fermionic operators as the product of the neutral fermions and the phases:

$$
\psi_{\sigma}(\tau, \mathbf{r})=\chi_{\sigma}(\tau, \mathbf{r}) \exp (i \theta(\tau, \mathbf{r}) / 2),
$$

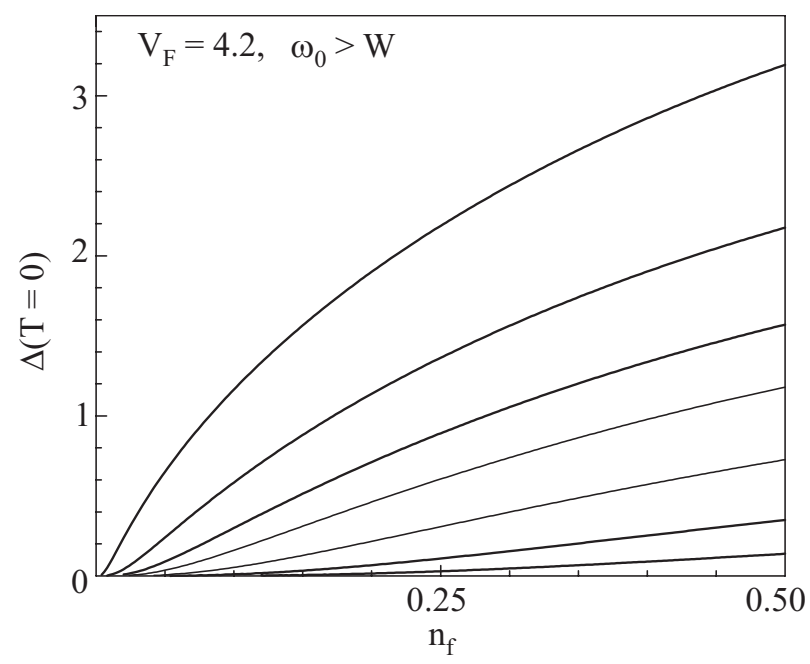

FIG. 2. The doping dependence of the zero-temperature gap at different values of coupling $V_{0}=3.2,4,5,6,6.8,8$, and 10 . 


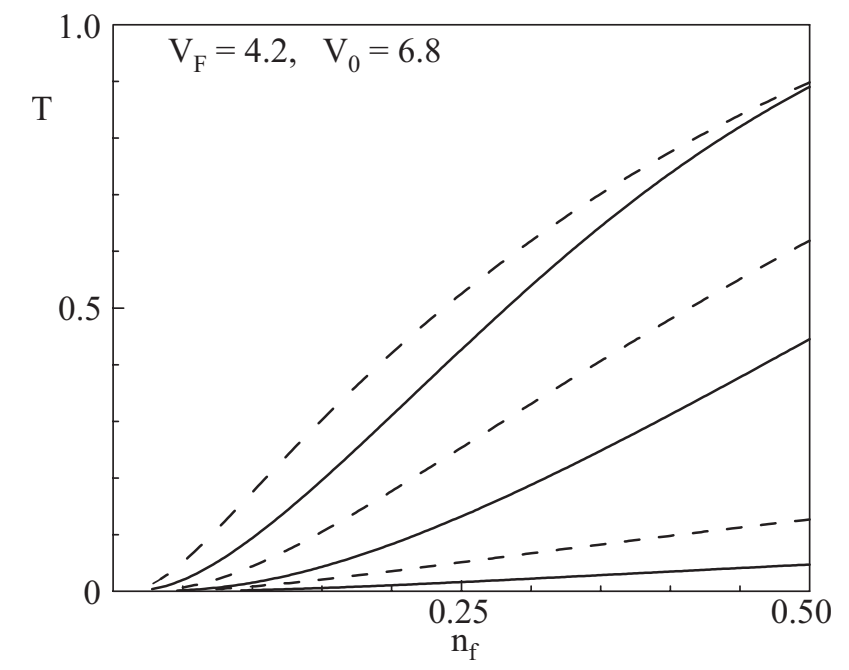

FIG. 3. The temperature-carrier density phase diagram at different values of frequency $\omega_{0}=1,5$, and 20 . The solid curves correspond to $T_{c}$ and the dashed curves to $T_{c}^{M F}$.

$$
\psi_{\sigma}^{\dagger}(\tau, \mathbf{r})=\exp (-i \theta(\tau, \mathbf{r}) / 2) \chi_{\sigma}^{\dagger}(\tau, \mathbf{r})
$$

such that

$$
\begin{aligned}
& \Psi(\tau, \mathbf{r})=\mathrm{e}^{i \tau_{z} \theta(\tau, \mathbf{r}) / 2} Y(\tau, \mathbf{r})=\mathrm{e}^{i \tau_{z} \theta(\tau, \mathbf{r}) / 2}\left(\begin{array}{c}
\chi_{\uparrow}(\tau, \mathbf{r}) \\
\chi_{\downarrow}^{\dagger}(\tau, \mathbf{r})
\end{array}\right), \\
& \Psi^{\dagger}(\tau, \mathbf{r})=Y^{\dagger}(\tau, \mathbf{r}) \mathrm{e}^{-i \tau_{z} \theta(\tau, \mathbf{r}) / 2}=\left(\begin{array}{c}
\chi_{\uparrow}^{\dagger}(\tau, \mathbf{r}), \\
\chi_{\downarrow}(\tau, \mathbf{r})
\end{array}\right) \mathrm{e}^{-i \tau_{z} \theta(\tau, \mathbf{r}) / 2} .
\end{aligned}
$$

In this case, the order parameters become

$$
\begin{aligned}
& \Phi\left(\tau, \mathbf{r}_{1}, \mathbf{r}_{2}\right)=\Delta\left(\tau, \mathbf{r}_{1}, \mathbf{r}_{2}\right) \exp (i \theta(\mathbf{R})), \\
& \Phi^{\dagger}\left(\tau, \mathbf{r}_{1}, \mathbf{r}_{2}\right)=\Phi^{*}\left(\tau, \mathbf{r}_{1}, \mathbf{r}_{2}\right)=\Delta\left(\tau, \mathbf{r}_{1}, \mathbf{r}_{2}\right) \exp (-i \theta(\mathbf{R})) .
\end{aligned}
$$

In the last equations, $\Delta\left(\tau, \mathbf{r}_{1}, \mathbf{r}_{2}\right)=\left|\Phi\left(\tau, \mathbf{r}_{1}, \mathbf{r}_{2}\right)\right|$ is the modulus of the order parameter, and $\theta(\mathbf{R}) \simeq\left[\theta\left(\mathbf{r}_{1}\right)+\theta\left(\mathbf{r}_{2}\right)\right] / 2$, where $\mathbf{R}=\left(\mathbf{r}_{1}+\mathbf{r}_{2}\right) / 2$ is the center-of-mass coordinate of two

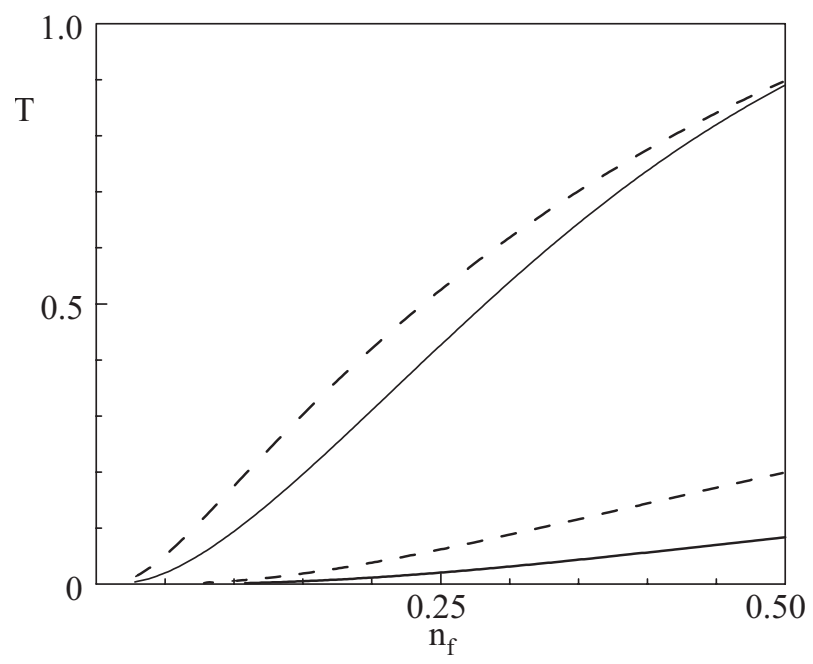

FIG. 4. The same as in Fig. 3 but for different values of the coupling, $V_{0}$ $=4,6.8$ and $v_{F}=4.2, \omega_{0}=20$. electrons, is its phase. This approximation corresponds to the limit of weak thermal fluctuations, when the space dependence of the phase is not strong. Below, we neglect the time (quantum) fluctuations, which become important only at very low temperatures, and also assume that the gap depends only on the relative electron coordinate $\mathbf{r}_{1}-\mathbf{r}_{2}$, i.e.,

$$
\Phi\left(\tau, \mathbf{r}_{1}, \mathbf{r}_{2}\right) \simeq \Delta\left(\mathbf{r}_{1}-\mathbf{r}_{2}\right) \mathrm{e}^{i \theta(\mathbf{R})} .
$$

The approximation given by Eq. (17) means that the dynamics of Cooper pairs is described by the order-parameter modulus, and its symmetry depends on the relative pair coordinate, whereas the motion of the superconducting condensate is defined by the slowly varying function $\theta(\mathbf{R})$, which depends on the center-of-mass coordinate of the electrons.

Substitution of Eqs. (15) and (17) into Eq. (7) and integration over the fermionic fields $\mathrm{Y}^{\dagger}$ and $\mathrm{Y}$ yield the following equation for the thermodynamic potential as a function of $\theta$ :

$$
\begin{aligned}
\Omega\left(\Delta, \partial_{\tau} \theta, \nabla \theta\right)= & T \int_{0}^{1 / T} d \tau \int d \mathbf{r}_{1} \int d \mathbf{r}_{2} \frac{1}{2} \frac{\left|\Delta\left(\mathbf{r}_{1}-\mathbf{r}_{2}\right)\right|^{2}}{V\left(\left(\mathbf{r}_{1}-\mathbf{r}_{2}\right)\right.} \\
& -\operatorname{Tr} \ln \left[G^{-1}-\Sigma\right]+\operatorname{Tr} \ln G^{-1}
\end{aligned}
$$

where

$$
G_{\mathbf{k}}\left(t, t^{\prime}\right)=-i\left\langle T\left(Y_{\mathbf{k}}(t) Y_{\mathbf{k}}^{\dagger}\left(t^{\prime}\right)\right\rangle\right.
$$

is the time-ordered mean-field matrix Nambu Green's function. It has the following form in the Matsubara frequencymomentum representation:

$$
G_{\mathbf{k}}\left(i \omega_{n}\right)=\frac{1}{i \omega_{n}-\tau_{z}(\varepsilon(\mathbf{k})-\mu)-\tau_{x} \Delta(\mathbf{k})} .
$$

In Eq. (18), $\Sigma$ is the self-energy of the inhomogeneous Green's function, which depends on the gradients of the phase of the order parameter:

$$
\begin{aligned}
\Sigma\left(\mathbf{r}_{1}, \mathbf{r}_{2}\right)= & \delta\left(\mathbf{r}_{1}-\mathbf{r}_{2}\right) \\
& \times\left[\mathrm{e}^{-i \tau_{z} \theta\left(\mathbf{r}_{1}\right) / 2} \tau_{z} \varepsilon\left(-i \nabla_{\mathbf{r}_{2}}\right) \mathrm{e}^{i \tau_{z} \theta\left(\mathbf{r}_{2}\right) / 2}-\tau_{z} \varepsilon\left(-i \nabla_{\mathbf{r}_{2}}\right)\right] .
\end{aligned}
$$

In Eq. (18), Tr means the space-time integration and the matrix trace (for details, see, e.g., Ref. 18). In order to find the thermodynamic potential, it is convenient to expand the logarithm in powers of $\Sigma$ :

$$
\operatorname{Tr} \ln \left[G^{-1}-\Sigma\right]=\operatorname{Tr} \ln G^{-1}+\operatorname{Tr} \sum_{n} \frac{1}{n}(G \Sigma)^{n} .
$$

This allows one to get the following expression for the second-order expansion of the thermodynamic potential in the limit of small fluctuations of the phase of the order parameter:

$$
\Omega(\Delta, \theta)=\frac{\mathcal{J}}{2} \int d^{2} r(\nabla \theta)^{2},
$$

where

$$
\mathcal{J}=\frac{v_{F}^{2}}{8 T} \int \frac{d^{2} k}{(2 \pi)^{2}} \frac{1}{\cosh ^{2}[E(\mathbf{k}) / 2 T]}
$$

is the superconducting stiffness (see Appendix). 
One can find the equation for the critical temperature of the BKT transition $T_{c}$, below which the phases of order parameter become algebraically ordered, by using the analogy with the 2D spin $X Y$ model, where the spin orientation corresponds to the vector $\nabla \theta$ in our problem. This equation has the following form:

$$
T_{c}=\frac{\pi}{2} \mathcal{J}\left(\Delta\left(\mu, T_{c}\right), \mu, T_{c}\right)
$$

where the function $\mathcal{J}$ is defined by Eq. (24). The doping, interaction, and bosonic frequency dependences of $T_{c}$ can be found by solving the system of equations (8), (9), and (25).

A numerical analysis shows that $T_{c}$ is significantly lower than $T_{c}^{M F}$ (Figs. 3 and 4). The critical temperature decreases with decreasing bosonic frequency (Fig. 3). When the coupling is not too strong, $T_{c}$ is exponentially suppressed (Fig. 4), similar to $T_{c}^{M F}$ and $\Delta(T=0)$. Our analysis shows that $T_{c}$ starts to grow rapidly at densities $n_{f}$ higher than the critical value $n_{f}^{\text {cr }} \sim 1 / V_{0}^{2}$ at large $\omega_{0}$. In particular, in this case in order to get a critical temperature higher than $1 \mathrm{~K}$, one needs to have the effective coupling $\sqrt{n_{f}} V_{0} \sim 1 \mathrm{eV}$ (see Ref. 14). It is possible that $T_{c}$ can be higher than the values obtained in this paper due to a van Hove singularity, if rather high values of doping $\left(\varepsilon_{F} \sim 3 \mathrm{eV}\right)$ can be obtained. We have found also that the ratio $2 \Delta(T=0) / T_{c}$ is bigger than the BCS value 3.52, and it increases with decreasing doping and acquires values approximately two times bigger those of BCS.

Besides the reduction of the value of the critical temperature, another important consequence of the thermodynamic fluctuations is a finite DOS in the gap region at temperatures $T_{c}<T<T_{c}^{M F}$, or the pseudogap phase, ${ }^{14}$ which can be observed experimentally in graphene systems. A similar phase is observed in high-temperature superconductors, where the thermal fluctuations may also be responsible for its formation (see, e.g., Ref. 19 and references therein).

\section{CONCLUSIONS}

We have studied the doping, coupling, and bosonfrequency dependences of the superconducting properties of a model of doped single-layer graphene by taking into account the thermodynamic fluctuations of the superconducting order parameter. We have shown that, due to the form of the doping dependence of the free-electron density of states, the superconductivity is suppressed at low doping, and at high values of doping the superconducting gap and critical temperature increase with doping growth, which is different from the two-dimensional system with the parabolic dispersion. We have found that the fluctuations lead to a significant reduction of the critical temperature. Namely, for realistic values of the model parameters, the critical temperature $T_{c}$ is exponentially suppressed at doping $n_{f}<n_{f}^{\mathrm{cr}} \sim\left(1 \mathrm{eV} / V_{0}\right)^{2}$ at large values of the bosonic frequency. The critical value $n_{f}^{\mathrm{cr}}$ decreases with decreasing $\omega_{0}$. The results obtained in the present paper can help to establish the mechanism of possible superconductivity in graphene, since the effective electron-electron coupling and the bosonic frequency can be estimated by comparing the theoretical results with the ex perimentally measured temperature-carrier density phase diagram.

V.M.L. acknowledges support through the Special Program of the NAS of Ukraine. V.T. thanks the Department of Energy for a partial support under grant number DOE-DEFG02-07ER15842.

\section{APPENDIX: THE THERMODYNAMIC POTENTIAL FOR THE PHASES OF THE ORDER PARAMETER}

In this Appendix we derive the expression for the lowenergy effective action for the phases of the superconducting order parameter, Eqs. (23) and (24).

In order to find the thermodynamic potential within the $(\nabla \theta)^{2}$ approximation, one needs to consider the first two terms in the expansion of Eq. (22):

$$
\operatorname{Tr} \ln \left[G^{-1}-\Sigma\right] \simeq \operatorname{Tr} \ln G^{-1}+\operatorname{Tr}[G \Sigma]+\frac{1}{2} \operatorname{Tr}[G \Sigma G \Sigma],
$$

where the $\theta$-dependent part of the self-energy given by Eq. (21) is

$$
\begin{aligned}
\Sigma\left(\mathbf{r}_{1}, \mathbf{r}_{2}\right)= & \delta\left(\mathbf{r}_{1}-\mathbf{r}_{2}\right) \\
& \times\left[\mathrm{e}^{-i \tau_{z} \theta\left(\mathbf{r}_{1}\right) / 2} \tau_{z} v_{F} \sqrt{-\nabla_{\mathbf{r}_{2}}^{2}} \mathrm{e}^{i \tau_{z} \theta\left(\mathbf{r}_{2}\right) / 2}-\tau_{z} v_{F} \sqrt{-\nabla_{\mathbf{r}_{2}}^{2}}\right]
\end{aligned}
$$

Since the last equation contains the square roots of the Laplace operators, it is more convenient to calculate the trace of the operators in Eq. (A1) in momentum space. In this case, the action of the operator $\sqrt{-\nabla_{\mathbf{r}}^{2}}$ has a simple form:

$$
\sqrt{-\nabla_{\mathbf{r}}^{2}} f(\mathbf{r})=\sum_{\mathbf{k}}|\mathbf{k}| \mathrm{e}^{i \mathbf{k r}} f_{\mathbf{k}}
$$

where $f_{\mathbf{k}}$ is the Fourier transform of $f(\mathbf{r})$. In the momentum representation, the effective action Eq. (23) has the following form:

$$
\Omega=\frac{J}{2} \sum_{\mathbf{k}} \mathbf{k}^{2} \theta_{\mathbf{k}} \theta_{-\mathbf{k}}
$$

Therefore, one needs to find the coefficient in front of $\theta_{-\mathbf{k}} \theta_{\mathbf{k}}$, which is equal to one-half of the the superconducting stiffness $\mathcal{J}$ in Eq. (23). Since the term linear in $\Sigma$ leads to a term proportional to $|\mathbf{k}| \theta_{\mathbf{k}}$ in Eq. (A1), we neglect it. In fact, the term linear in $\theta$ can be absorbed into the bilinear term by defining a new phase $\theta$ shifted by the corresponding factor. Therefore, we consider only the second-order term:

$$
\frac{1}{2} \operatorname{Tr}[G \Sigma G \Sigma]=-\frac{v_{F}^{2}}{8} \operatorname{Tr}\left[\sum_{\mathbf{p}} G_{\mathbf{p}}^{2}\right] \sum_{\mathbf{k}} \mathbf{k}^{2} \theta_{\mathbf{k}} \theta_{-\mathbf{k}},
$$

which gives

$$
J=-\frac{v_{F}^{2} T}{4} \operatorname{Tr} \sum_{\mathbf{p}}\left[G_{\mathbf{p}}^{2}\right] .
$$

In the derivation of Eq. (A5) we kept only the term propor- 
tional to $\mathbf{k}^{2} \theta_{\mathbf{k}} \theta_{-\mathbf{k}}$. Equation (A5) gives the expression for the superconducting stiffness (24).

${ }^{\text {a)} E-m a i l: v l o k t e v @ b i t p . k i e v . u a ~}$

${ }^{1}$ A. H. Castro Neto, F. Guinea, N. M. R. Peres, K. S. Novoselov, and A. K. Geim, Rev. Mod. Phys. (in press); preprint arXiv:0709.1163v2..

${ }^{2}$ Y. Kopelevich, P. Esquinazi, J. H. S. Torres, and S. Moehlecke, J. Low Temp. Phys. 119, 691 (2000).

${ }^{3}$ R. R. da Silva, J. H. S. Torres, and Y. Kopelevich, Phys. Rev. Lett. 87, 147001 (2001).

${ }^{4}$ S. Moehlecke, Y. Kopelevich, and M. B. Maple, Phys. Rev. B 69, 134519 (2004).

${ }^{5}$ Y. Kopelevich, S. Moehlecke, and R. R. da Silva, in Carbon Based Magnetism, T. Makarova and F. Palacio (eds.), Elsevier Science (2006), Chap. 18.

${ }^{6}$ B. Uchoa, G. G. Cabrera, and A. H. Castro Neto, Phys. Rev. B 71, 184509 (2005).
${ }^{7}$ E. Zhao and A. Paramekanti, Phys. Rev. Lett. 97, 230404 (2006).

${ }^{8}$ E. C. Marino and Lizardo H. C. M. Nunes, Nucl. Phys. 741, 404 (2006); Physica (Amsterdam) 460C-462C, 1101 (2007); Nucl. Phys. B 769, 275 (2007).

${ }^{9}$ B. Uchoa and A. H. Castro Neto, Phys. Rev. Lett. 98, 146801 (2007).

${ }^{10}$ M. Black-Schaffer and S. Doniach, Phys. Rev. B 75, 134512 (2007).

${ }^{11}$ C. Honerkamp, Phys. Rev. Lett. 100, 146404 (2008).

${ }^{12}$ N. B. Kopnin and E. B. Sonin, Phys. Rev. Lett. 100, 246808 (2008).

${ }^{13}$ J. Gonzales, Phys. Rev. B 78, 205431 (2008).

${ }^{14}$ V. M. Loktev and V. M. Turkowski, Phys. Rev. Lett. 79, 233402 (2009).

${ }^{15}$ V. M. Loktev and V. M. Turkowski, JETP 87, 329 (1998).

${ }^{16}$ V. M. Loktev, S. G. Sharapov, and V. M. Turkowski, Physica C 296, 84 (1998).

${ }^{17}$ V. M. Loktev and V. M. Turkowski, Fiz. Nizk. Temp. 30, 247 (2004) [Low Temp. Phys. 30, 179 (2004)].

${ }^{18}$ S. G. Sharapov, H. Beck, and V. M. Loktev, Phys. Rev. B 64, 134519 (2001).

${ }^{19}$ P. A. Lee, N. Nagaosa, and X. G. Wen, Rev. Mod. Phys. 78, 17 (2006).

${ }^{20}$ V. M. Loktev, R. M. Quick, and S. G. Sharapov, Phys. Rep. 349, 1 (2001).

This article was published in English in the original Russian journal. Reproduced here with stylistic changes by AIP. 